\title{
An optical and near-infrared exploration of the star formation region in Cygnus surrounding RNO 127 ${ }^{\star}$
}

\author{
T. Movsessian ${ }^{1,3}$, T. Khanzadyan ${ }^{2,1}$, T. Magakian ${ }^{1,3}$, M. D. Smith ${ }^{4}$, and E. Nikogosian ${ }^{1,3}$ \\ 1 Byurakan Astrophysical Observatory, 378433 Aragatsotn reg., Armenia \\ e-mail: tigmov@bao.sci.am \\ 2 Max-Planck Institut für Astronomie, Königstuhl 17, 69117 Heidelberg, Germany \\ e-mail: khtig@mpia-hd.mpg.de \\ 3 Isaac Newton Institute of Chile, Armenian Branch \\ e-mail: tigmag@sci.am \\ 4 Armagh Observatory, College Hill, Armagh BT61 9DG, Northern Ireland, UK \\ e-mail:mds@star.arm.ac.uk
}

Received 8 April 2003 / Accepted 22 August 2003

\begin{abstract}
We investigate a relatively unstudied star formation region in Cygnus centered on RNO 127, finding numerous Herbig-Haro flows, many identified in optical [SII], $\mathrm{H} \alpha$, and near-infrared $\mathrm{H}_{2}$ tracers of shock waves. Several protostars and young stars are thus located, including one conspicuously brightened object, which illuminates a variable reflection nebula. In total, the coordinates of 17 optical HH knots and jets, 4 associated cometary nebulae and 3 NIR objects are given. Individual structures are discussed including a central complex which has the characteristics of superposed HH flows. This star-forming cloud is not isolated but is part of a much larger region of distributed star formation, including $\mathrm{HH} 380$ and $\mathrm{HH} 381$.
\end{abstract}

Key words. stars: formation - ISM: jets and outflows - ISM: clouds

\section{Introduction}

Several star-forming regions with high concentrations of stars and widespread outflow activity have been found in recent years (Reipurth \& Bally 2001). Individual outflows often possess high collimation, as revealed by Herbig-Haro flows, bipolar outflows, maser distributions and cometary nebula. Furthermore, near-infrared jets and bullets of $\mathrm{H}_{2}$ emission and (sub)-millimetre cores of high luminosity are also common. The energy for all these phenomena are believed to be derived from high gas accretion rates (Motte \& André 2001; Beuther et al. 2002), characteristic of the earliest stages of star formation.

Here we present an investigation of a relatively unstudied star formation region in which optical observations have uncovered an extensive distribution of Herbig-Haro objects. Studies of such regions assist progress on many issues such as how

Send offprint requests to: T. Khanzadyan,

e-mail: khtig@mpia-hd.mpg.de

* Based on observations collected at the Byurakan Astrophysical Observatory, Byurakan, Armenia. Includes observations made at German-Spanish Astronomical Centre, Calar Alto, operated by the Max-Planck-Institut für Astronomie. star formation is initiated in different locations and how it then evolves.

During an ongoing imaging survey of nebulous objects in dark clouds, conducted at the Byurakan Observatory, we found one particular field with numerous optical manifestations of star formation. Combining wide-field optical and near-infrared observations, we here investigate the structure of this region, as well as its relationship to other neighbouring clouds, and the evolution of the region as a whole. In the center of this field lies RNO 127 (Cohen 1980), around which we investigated a wide area ( 14 square arcmin). This object is also known as HH $448^{1}$ (Melikian \& Karapetian 2001). Nearby, we have found a striking variable cometary nebula and several other Herbig-Haro objects. A number of IRAS sources were also found to lie in this area leading us to suspect that this region must be one of active star formation and hence, to extend our investigation.

To the west lie several IRAS sources as well as three catalogued Herbig-Haro objects (HH 380, HH 381 and HH 382), which form at least two outflows (Devine et al. 1997). HH 380

1 The name HH448 was reserved by N. Melikian for a new HH-like object, which is identical to RNO 127, but was not used in their article (Melikian \& Karapetian 2001) (from priv. comm. N. Melikian and B. Reipurth). 
Table 1. Log of the observations.

\begin{tabular}{lccccrr}
\hline \hline Band name & $\lambda_{\mathrm{c}}$ & $\Delta_{\lambda}$ & Covered area & Pixel scale & Seeing & Observing dates \\
\hline$[$ SII] & $6730 \AA$ & $75 \AA$ & $11^{\prime} \times 5.5^{\prime}$ & $0.65^{\prime \prime}$ & $1.5^{\prime \prime}-2.0^{\prime \prime}$ & 2000. Aug. 22, 25 \\
$\mathrm{H}_{\alpha}$ & $6560 \AA$ & $85 \AA$ & $11^{\prime} \times 5.5^{\prime}$ & $0.65^{\prime \prime}$ & $1.5^{\prime \prime}-2.0^{\prime \prime}$ & 2000. Aug. 22, 25 \\
$I$-band & $8500 \AA$ & $1200 \AA$ & $11^{\prime} \times 5.5^{\prime}$ & $0.65^{\prime \prime}$ & $1.5^{\prime \prime}-2.0^{\prime \prime}$ & 2000. Aug. 22, 25 \\
{$[$ SII] } & $6730 \AA$ & $75 \AA$ & $14^{\prime} \times 14^{\prime}$ & $0.42^{\prime \prime}$ & $1.5^{\prime \prime}-2.0^{\prime \prime}$ & 2001. Nov. 19 \\
$\mathrm{H}_{\alpha}$ & $6560 \AA$ & $85 \AA$ & $14^{\prime} \times 14^{\prime}$ & $0.42^{\prime \prime}$ & $1.5^{\prime \prime}-2.0^{\prime \prime}$ & 2001. Nov. 19 \\
$I$-band & $8500 \AA$ & $1200 \AA$ & $14^{\prime} \times 14^{\prime}$ & $0.42^{\prime \prime}$ & $1.5^{\prime \prime}-2.0^{\prime \prime}$ & 2001. Nov. 19 \\
{$[$ SII] } & $6730 \AA$ & $75 \AA$ & $14^{\prime} \times 14^{\prime}$ & $0.42^{\prime \prime}$ & $1.5^{\prime \prime}-2.0^{\prime \prime}$ & 2002. July. 31 \\
$\mathrm{H}_{\alpha}$ & $6560 \AA$ & $85 \AA$ & $14^{\prime} \times 14^{\prime}$ & $0.42^{\prime \prime}$ & $1.5^{\prime \prime}-2.0^{\prime \prime}$ & 2002. July. 31 \\
$I$-band & $8500 \AA$ & $1200 \AA$ & $14^{\prime} \times 14^{\prime}$ & $0.42^{\prime \prime}$ & $1.5^{\prime \prime}-2.0^{\prime \prime}$ & 2002. July. 31 \\
$\mathrm{H}_{2} 1-0 \mathrm{~S}(1)$ & $2.125 \mu \mathrm{m}$ & $0.02 \mu \mathrm{m}$ & $7^{\prime} \times 7^{\prime}$ & $0.396^{\prime \prime}$ & $1.0^{\prime \prime}-1.6^{\prime \prime}$ & 2000. Dec. 09 \\
$K_{\mathrm{s}}$-band & $2.196 \mu \mathrm{m}$ & $0.39 \mu \mathrm{m}$ & $12^{\prime} \times 8^{\prime}$ & $0.396^{\prime \prime}$ & $1.5^{\prime \prime}-1.9^{\prime \prime}$ & 2000. Dec. 09 \\
\hline
\end{tabular}

is $11^{\prime}$ west from of RNO 127 , a projected distance of just $2.7 \mathrm{pc}$. It is possible that the two regions of star formation are related. They are located within a Cygnus OB7 complex (see Dobashi et al. 1996, with galactic coordinates $l \sim 92^{\circ}$, $b \sim 3^{\circ}$ ), therefore a distance of $d=800 \mathrm{pc}$ is adopted for the investigated region.

\section{Observations and data reduction}

\subsection{Optical observations}

Observations were carried out with the 2.6-meter telescope of the Byurakan Observatory during five campaigns between 2000 and 2002. First optical images were obtained in August 2000 with the ByuFOSC-2 (Movsessian et al. 2000) camera attached at the prime focus. A Thomson $1060 \times 1028 \mathrm{CCD}$ detector was used working in half obscured mode with a real image size of $1060 \times 514$ pixel (see Movsessian et al. 2000, for mode explanation). The field of view was $11.5^{\prime} \times 5.5^{\prime}$ with a pixel size of $0.65^{\prime \prime}$. In 2001-2002 the SCORPIO ${ }^{2}$ camera working with a Lick $2063 \times 2058$ pixel CCD was used. This new camera provides a field of view of $14^{\prime} \times 14^{\prime}$ with $0.42^{\prime \prime}$ sampling. During the observations, the mean seeing was $1.5^{\prime \prime}-2^{\prime \prime}$. The optical images were taken through [SII] $\left(\lambda_{\mathrm{c}}=6730 \AA, \Delta_{\lambda}=75 \AA\right)$ and $\mathrm{H} \alpha\left(\lambda_{\mathrm{c}}=6560 \AA, \Delta_{\lambda}=85 \AA\right)$ interference filters and, for the continuum, an $I$-band filter $\left(\lambda_{\mathrm{c}}=8500 \AA, \Delta_{\lambda}=1200 \AA\right)$ was used.

Table 1 summarises the optical observations. The data reduction was done with the ESO-MIDAS ${ }^{3}$ image processing system and with locally developed packages.

${ }^{2}$ SCORPIO is Spectral Camera with Optical Reducer for Photometrical and Interferometrical Observations build at the Special Astrophysical Observatory, Russia under scientific project advisor of V. L. Afanasiev.

${ }^{3}$ ESO-MIDAS is the acronym for the European Southern Observatory Munich Image Data Analysis System which is developed and maintained by the European Southern Observatory.

\subsection{Near infrared observations}

The near infrared observations of this region were carried out December 9, 2000 using Omega Prime (Bizenberger et al. 1988) at the Calar Alto $3.5 \mathrm{~m}$ telescope. Omega Prime is equipped with a Rockwell $1024 \times 1024$ pixel HAWAII array detector. It provides a pixel scale of $0.396^{\prime \prime} /$ pixel scale and a total field of view of $6.8^{\prime} \times 6.8^{\prime}$.

Images were obtained in the narrow band $\mathrm{H}_{2} 1-0 \mathrm{~S}(1)$ filter centered at $\lambda=2.125 \mu \mathrm{m}$ and a width of $0.0206 \mu \mathrm{m}$. Since the camera was not equipped with an appropriate narrow band continuum filter, the $K_{\mathrm{s}}$ broadband filter, with passband centered at $\lambda=2.196 \mu \mathrm{m}$, was alternatively used for continuum subtraction. Observations were done using Full MPIA double correlated read mode (Bizenberger et al. 1988), which has a minimum detector integration time (DIT) of $1.677 \mathrm{~s}$. By using this method for both filters, each "individual frame" was constructed from 47 co-added DITs resulting in about $79 \mathrm{~s}$ of integration. In $K_{\mathrm{s}} 9$ frames were taken and in $\mathrm{H}_{2}-14$ frames with standard 5-point jittering.

Table 1 summarises the near-infrared observations. Data reduction proceeded through standard routines using CCDPACK and KAPPA data reduction packages developed under the Starlink Project: i.e. the images were dark subtracted, flat fielded, sky subtracted and then combined to form a mosaic picture of the complete field. Flat field calibrations were achieved via dome flats using difference frames of integrations with a halogen lamp on and off.

The next step was to identify pure $\mathrm{H}_{2} 1-0 \mathrm{~S}(1)$ line emission objects by subtracting the continuum. This was done by subtracting the $K_{\mathrm{s}}$ broad band image from $\mathrm{H}_{2}$ 1-0 S(1) line image according to the scheme $\mathrm{H}_{2} \times$ Const. $-K_{\mathrm{s}}$, where the Const. value was determined from the difference in filter widths and from the total integrations in both filters. In this particular case, the $K_{\mathrm{s}}$ band filter is 20.4 times wider than $\mathrm{H}_{2} 1-0 \mathrm{~S}(1)$ line filter. Then by multiplying this value by ratio of the total integrations in both filters $(710 \mathrm{~s} / 1100 \mathrm{~s})$ we get Const. $=13.2$. This value was used for the first approximate continuum subtraction. Further "tuning" of the Const. value was achieved (Const. $=12.5$ ) by changing the value in order to subtract stars in the field. Because this method of continuum subtraction is 


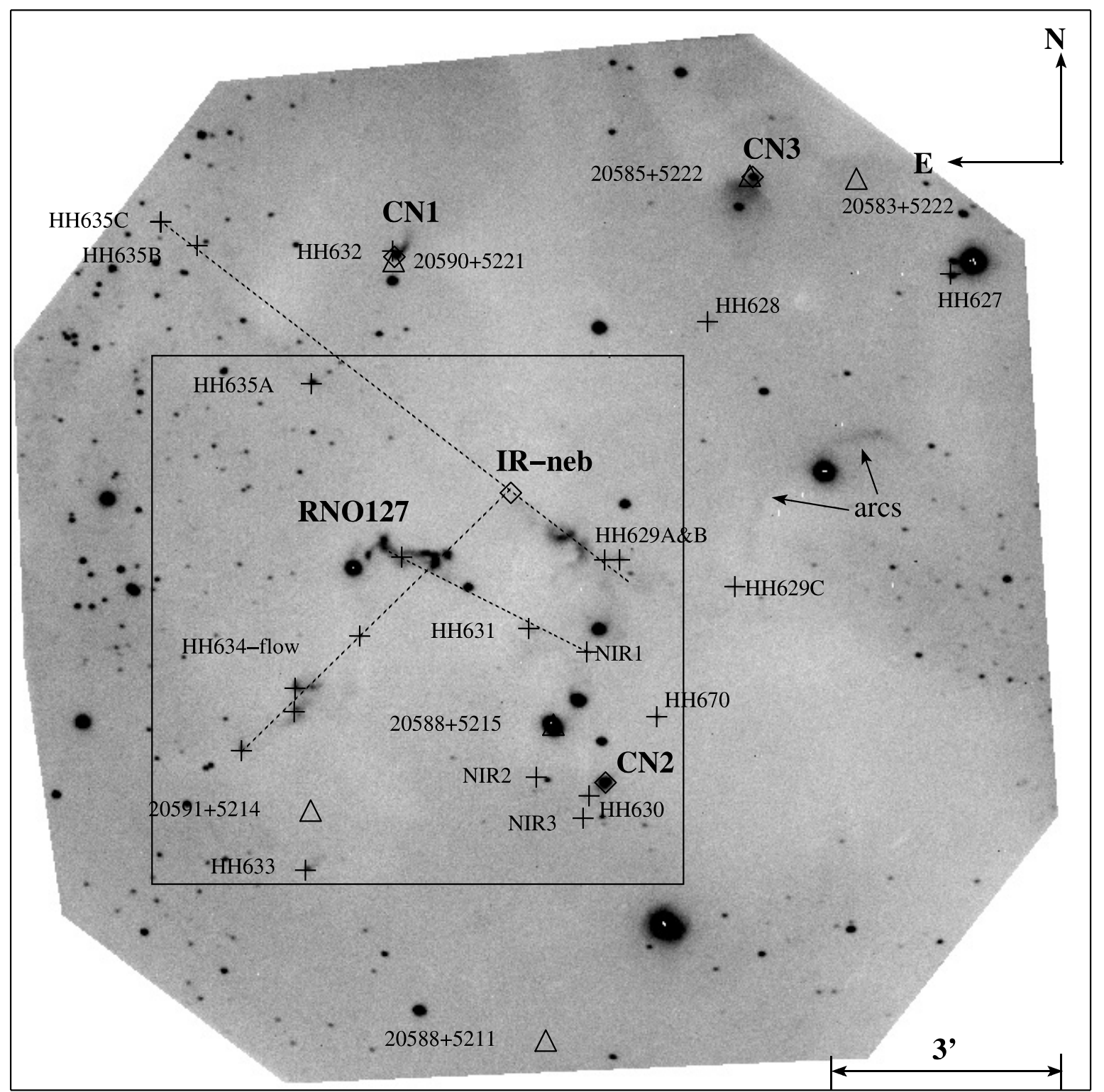

Fig. 1. $\mathrm{H} \alpha+[\mathrm{SII}]$ image of the observed region. North is up and east is left. The inner box indicates the coverage of the near-infrared observations presented by Fig. 2. The scale is indicated in right bottom corner. All newly discovered HH objects are marked on the picture with crosses and described in Table 2. IRAS objects are marked with triangles and listed in Table 3. Diamonds indicate the positions of nebulae, which are explained in the text. Dotted lines indicate the suggested flows explained in the text.

not accurate, we can only state the presence and morphology of found objects.

\section{Results and discussion}

\subsection{Structure of the field}

The $\mathrm{H} \alpha+[\mathrm{SII}]$ composite image of the observed region in Cygnus is shown in Fig. 1. The inner box indicates the coverage of near-infrared observations (see Fig. 2). The new HH objects are marked on the image with "+" signs and presented in Table 2. The first column of Table 2 lists their names while the second and third columns give the coordinates. The fourth column indicates the notes on each object, as explained below the table.
Figure 2 shows the region of Cygnus covered by the near infrared $\left(\mathrm{H}_{2} 1-0 \mathrm{~S}(1)\right)$. The newly discovered $\mathrm{H}_{2}$ emission objects as well as infrared reflection nebulae are marked on the image in addition to the newly found optical knots.

As is obvious from these images, although RNO 127 and nearby IR nebulae dominate in the field, it is rich in $\mathrm{HH}$ objects, which form several probable flows. We discuss below these flows and their proposed sources in more detail. Note that RNO 127 lies in the densest part of the cloud, where no background stars are seen even in the NIR image. The extinction appears to extend over a $6^{\prime}$ region in the optical but only $3^{\prime}$ in the near-infrared. This may partly explain why $\mathrm{HH}$ objects are detectable at both wavelengths in the cloud outskirts.

There are also arc-like emission structures (see Fig. 1) extending from the bright star $\sim 5^{\prime}$ west of RNO 127. Their 
Table 2. New objects discovered in the optical and near-infrared.

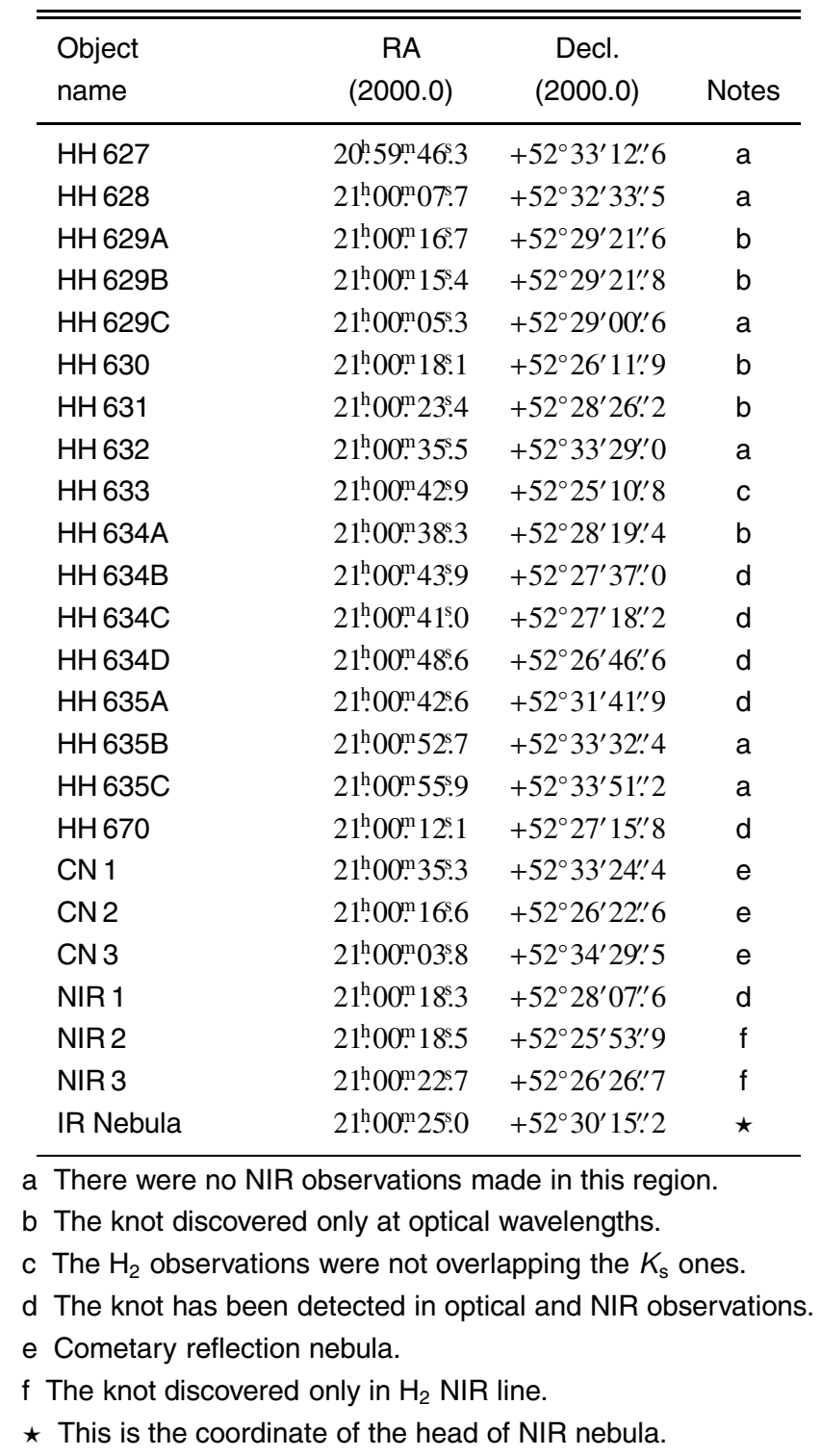

origin is not clear but the structures resemble that of the "Orion streamers" (Reipurth \& Sandell 1985).

We find that 6 IRAS sources are located in the studied field. Their properties are listed in Table 3, where the first column lists the object names; second to fourth columns give flux value in Janskys for four IRAS bands. The fifth to seventh columns detail the error ellipse of IRAS source positions; the major axis $\left(a^{\prime \prime}\right)$ the minor axis $\left(b^{\prime \prime}\right)$ of the error ellipse $\left(\mathrm{PA}^{\circ}\right)$, and the position angle (east of north) of the error ellipse (Beichman et al. 1988). The ninth column lists the other names of IRAS sources or otherwise the objects which have almost identical coordinates as the IRAS object. Particularly two of them are associated with small reflection nebulae (CN3 and CN1); one is associated with a T Tau type star (GLMP1017, Garcia-Lario et al. 1997); and one is associated with an $\mathrm{H} \alpha$ object (Cyg21, Melikian et al. 1996). The last column in Table 3 gives the names of objects or regions which are associated with each IRAS source according to the "IRAS Catalogue of Point Sources version 2.0 (IPAC 1996) Associations" by the Joint IRAS Science Working Group (1988).

Figure 3 is a DSS2-Infrared ${ }^{4}$ Survey extended view of our investigated region in Cygnus and the neighbouring region, which contains HH380 and HH381. The contours are from IRAS HiRes ${ }^{5} 100 \mu \mathrm{m}$ image, requested from IPAC ${ }^{6}$. Newly found and previously known objects are marked in the figure (see caption of Fig. 3 for details).

From this figure one can see the positions of nearby molecular clouds. To the south-east of RNO 127 is LDN1004 $\left(\sim 11^{\prime}\right)$ while to the south-west lie LDN1003 $\left(\sim 12^{\prime}\right)$ and [DBY94] 091.5+04.4 ( 24'). All are part of the extended Cygnus OB7 complex at a distance of 800 pc (Dobashi et al. 1994). It is logical therefore to adopt this distance for the RNO 127 field.

\subsection{HH objects}

\subsection{1. $\mathrm{HH} 627$ and $\mathrm{HH} 670$}

HH 627 is a small star-like HH object about $20^{\prime \prime}$ south-east of the bright $(B=12.4)$ star in the north-west of the optical field (see Fig. 1). There is no evidence that this bright star is the source of HH 627.

HH 670 is also a small star-like HH knot located about $1^{\prime}$ from the $\mathrm{CN} 2$ cometary nebula. With the present data we were not able to suggest any association of this knot with other knots or flows.

\subsection{2. $\mathrm{HH} 629$}

This is a chain of faint HH objects. Knots HH 629 A and B are located near the axis of the IR nebula in the south-western direction as indicated in Figs. 1 and 2 with dotted lines; HH 629 C is slightly aside. If these objects are really connected with the IR nebula, then it is logical to assume that they are directed towards us. This assumption is based on the simple fact that the IR-nebula has its visible side inclined towards the observer, but one should obtain more observational evidence before stating this fact explicitly.

\subsection{3. $\mathrm{HH} 631$ and NIR 1}

HH 631 has a diffuse elongated appearance towards the northeast. In about $50^{\prime \prime}$ towards the south-west lies the NIR 1 starlike knot which is detected only in the $\mathrm{H}_{2} \mathrm{~S}(1-0)$ near-infrared

\footnotetext{
${ }^{4}$ DSS2-Infrared is part of the POSS-II, made by Caltech with funds from the NSF, the NG Society, the Sloan Foundation, the Samuel Oschin Foundation, and the Eastman Kodak Corporation.

${ }^{5}$ HiRes uses the Maximum Correlation Method (MCM, Aumann et al. 1990) to produce images with better than nominal resolution of the Infrared Astronomical Satellite (IRAS) data.

${ }^{6}$ IPAC is Infrared Processing and Analysis Center established as the NASA archival center for the IRAS data and operated by the JPL and California Institute of Technology (Caltech) http://www.ipac.caltech.edu
} 


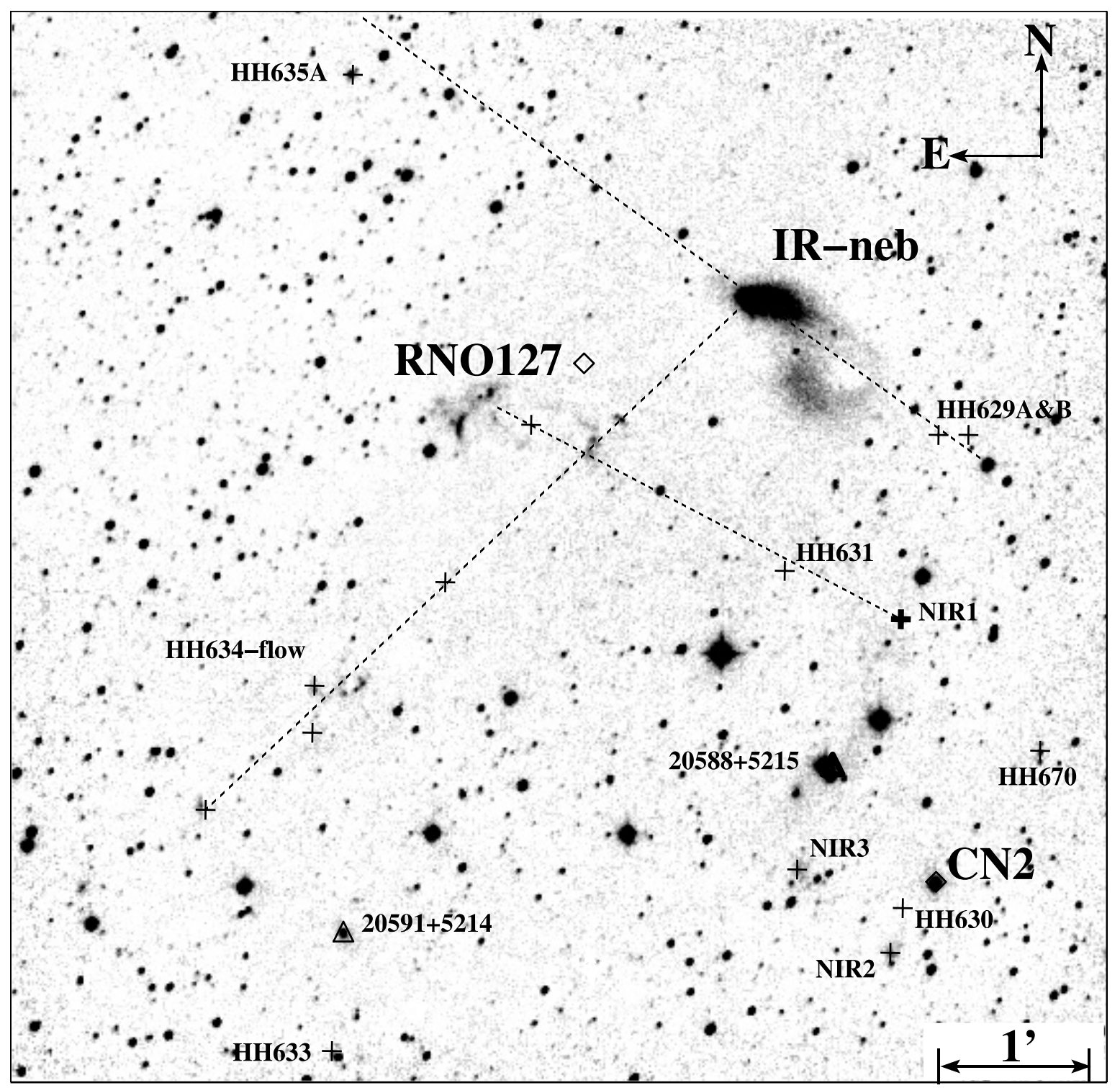

Fig. 2. Part of the Cygnus region in $\mathrm{H}_{2} 1-0 \mathrm{~S}(1)$ line + Continuum. North is up and east is left. Scale is indicated in the right bottom corner. Objects are marked as in Fig. 1. Dotted lines show suggested flows.

Table 3. Properties of the IRAS sources close to RNO 127 as labeled in Fig. 1. The flux unit is Jansky. See text for explanations.

\begin{tabular}{lcccccccrc}
\hline \hline IRAS object & $12 \mu \mathrm{m}$ & $25 \mu \mathrm{m}$ & $60 \mu \mathrm{m}$ & $100 \mu \mathrm{m}$ & $a\left[^{\prime \prime}\right]$ & $b\left[^{\prime \prime}\right]$ & $\mathrm{PA}\left[^{\circ}\right]$ & Other names & Assoc. obj. \\
\hline $20583+5222$ & 0.25 & 0.38 & 2.30 & 9.8 & 29 & 9 & 45 & X2058+523 \\
$20585+5222$ & 0.36 & 0.46 & 1.52 & 11.2 & 13 & 5 & 138 & CN 3 & X2058+523 \\
$20588+5211$ & 0.29 & 0.46 & 0.65 & 7.0 & 9 & 9 & 153 & LDN1003 \\
$20588+5215$ & 0.64 & 2.98 & 8.22 & 9.5 & 7 & 5 & 25 & GLMP 1017 & LDN1003 \\
$20590+5221$ & 0.36 & 0.27 & 0.85 & 10.7 & 25 & 10 & 137 & CN 1 & X2058+523 \\
$20591+5214$ & 0.27 & 0.27 & 1.14 & 7.5 & 11 & 9 & 150 & [MKH96]Cyg21 & LDN1004 \\
\hline
\end{tabular}

line ${ }^{7}$. Their connecting line towards the north-east crosses the knot $\mathrm{C}$ in RNO 127(HH448) (see Fig. 4) and a faint appendage to the south-west of RNO 127B. This direction is marked in
Fig. 4 as one of the possible intersecting outflows, which contribute to the RNO 127 morphology (see 3.2.4). Thus the relation of these objects to RNO 127 cannot be excluded.

7 During the revision of our manuscript we have detected also weak optical counterpart of NIR 1 object in [SII] emission. 


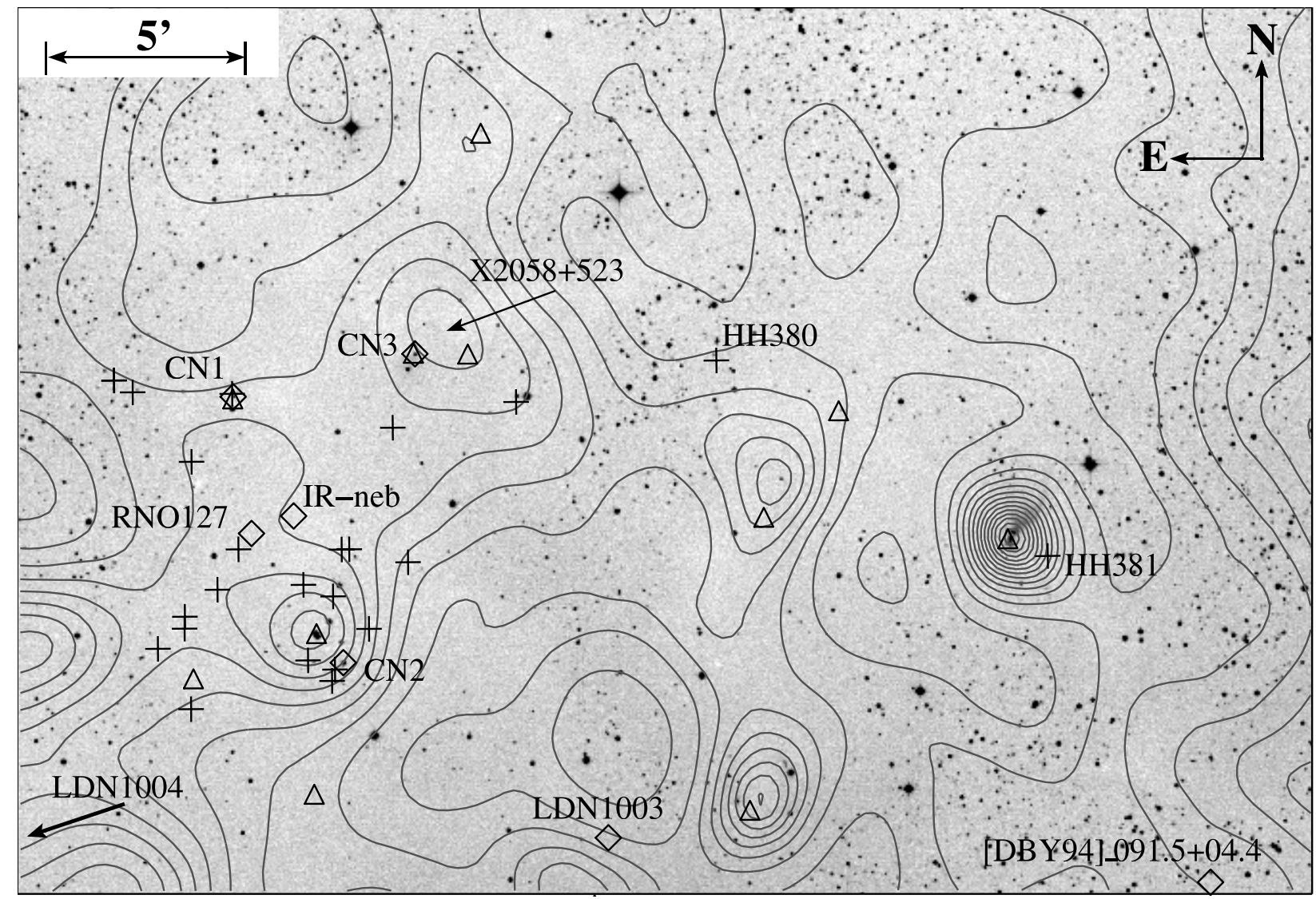

Fig. 3. DSS2-Infrared Survey image of the extended region in Cygnus $\left(34^{\prime} \times 23^{\prime}\right)$ with overlaid IRAS HiRes $100 \mu$ m contours. North is up and East is left. The scale is indicated in the left upper corner. Positions of new and known HH objects are marked with "+" signs; the IRAS sources are marked with triangles; the nebulae and dark clouds are marked with diamonds.

\subsubsection{RNO 127}

In our investigation of RNO 127, we were able to detect emission in the optical and near-infrared lines (see Fig. 2 and Table 2). Figure 4 displays a close-up of RNO 127 in the $\mathrm{H}_{2}$, [SII] and $\mathrm{H} \alpha$ lines. The $\mathrm{H}_{2}$ image was binned in order to align with the $[\mathrm{SII}]$ and $\mathrm{H} \alpha$ band pictures.

The upper right panel of Fig. 4 shows RNO 127 in continuum subtracted $\mathrm{H}_{2}$ emission (as explained in Sect. 2.2), where the arrows indicate the suggested flow directions. The upper left panel shows RNO 127 in [SII] emission and the the lower left panel shows $\mathrm{H} \alpha$ emission. The lower right panel displays the $\mathrm{H} \alpha /[\mathrm{SII}]$ ratio image with the color bar indicating the the range of ratio values.

RNO 127 consist of four distinctive knots (A, B, C and D) seen in all observed bands, situated within $50^{\prime \prime}$. From close examination one can see its complex structure which could be the result of several interacting or spatially superimposed outflows, as suggested by the arrows in the upper right panel of Fig. 4.

One of the suggested directions, "from HH 634", coincides well with the HH 634-flow (see dotted line in Figs. 1 and 2 and Sect. 3.2.6). There are two knots (A \& B) which lie on the line connecting the HH634-flow with RNO 127. Both knots have nearly equal strength in $\mathrm{H} \alpha$, wheres in the [SII] line knot $\mathrm{A}$ is much fainter (as seen in Fig. 4).
Knot A may be a bow-like structure in a flow from the South-East direction. It is distinguished by its higher atomic excitation level, revealed in the lower right panel of Fig. 4. An arc-shape is also observed in $\mathrm{H}_{2}$, with strong emission from the projected bow apex. This suggests that the flow is far from the plane of the sky, allowing the $\mathrm{H}_{2}$ to appear on a limb-brightened section of the bow wing and the optical emission to peak on the bow apex, offset in projection from the leading edge (as, for example, occurs in HH 7 (Smith et al. 2003)).

Another possible flow ("from HH 631") contributing to the shaping of the RNO 127 structure is from the south-west (see Figs. 1 and 2). Knot $\mathrm{C}$ and knot $\mathrm{D}$ both have a bow-like appearance and are embedded in a common diffuse structure. Knot B and the elongated diffuse feature could represent another part of this outflow.

The last arrow (marked as "?") suggests possible third flow on the basis of the bow-like structure of knot $\mathrm{D}$ seen in $\mathrm{H}_{2}$ line. But no suitable object has been found in this direction. It would be that HH 631 flow can also account for the origin of knot D. It is quite possible that the flow from HH 631 has some small opening angle with precession, which can produce multiple bow-shock like structures in one flow as is described in the HAMMER-jet models (Suttner et al. 1997; Smith et al. 1997a).

Finally, in the lower right panel of Fig. 4 we show the $[\mathrm{SII}] / \mathrm{H} \alpha$ ratio image of RNO 127 . The colour-bar indicates the value of the ratio which is proportional to the ionization 


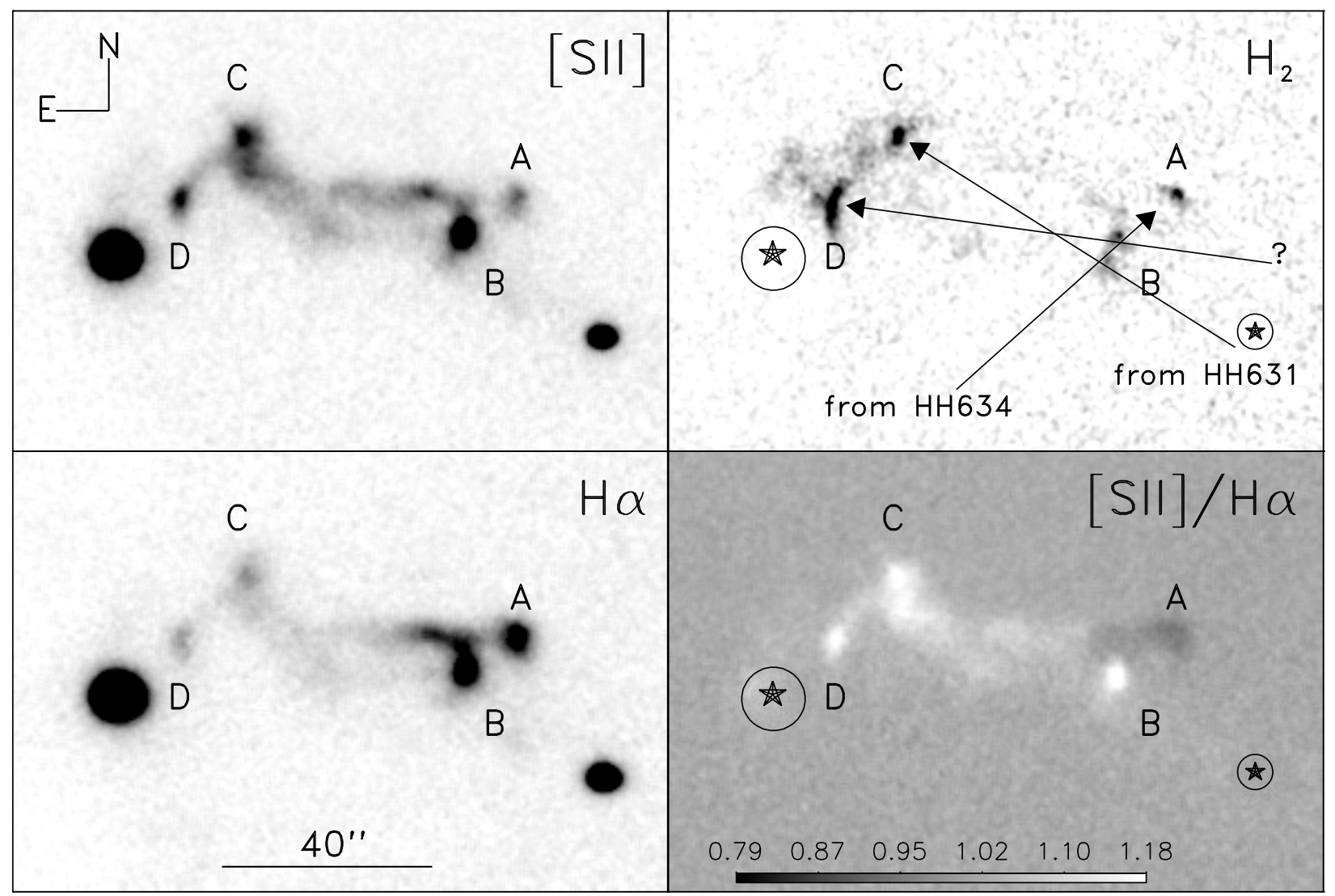

Fig. 4. RNO 127 (HH 448) in optical and near-infrared emission. North is up and east is left. Upper left panel shows the object in the [SII] emission line; upper right panel is pure $\mathrm{H}_{2} 1-0 \mathrm{~S}(1)$ emission from RNO 127 with arrows suggesting directions of 3 different flows, stars are subtracted during continuum subtraction and therefore are marked on the panel; the lower right panel is emission in $\mathrm{H} \alpha$ line and the lower right panel is $[\mathrm{SII}] / \mathrm{H} \alpha$ ratio image with colour-bar indicating values (see Sect. 3.2.4 for more explanation)

fraction of the shocked material. The $[\mathrm{SII}] / \mathrm{H} \alpha$ ratio can be directly compared to the model presented by Hartigan et al. (1994) in order to estimate the shock velocities in the different parts of RNO 127 (Fig. 9 of Hartigan et al. 1994). The observed $[\mathrm{SII}] / \mathrm{H} \alpha$ ratio of 1.18 corresponds to the speed of $\sim 40 \mathrm{~km} \mathrm{~s}^{-1}$ and the ratio of 0.79 corresponds to the speed of $\sim 50 \mathrm{~km} \mathrm{~s}^{-1}$. The difference in velocities, however, is not substantial $\left(\leq 10 \mathrm{~km} \mathrm{~s}^{-1}\right)$ and the idea of two (or more) separate outflows needs to be tested with additional spectral and proper motion studies. The fact that we have a very complex structure does not necessitate that the outflows are superimposed but an interaction would also account for the unusual morphology.

\subsection{5. $\mathrm{HH} 633$}

A composite image of HH633 and IRAS 20591+5214 in the [SII] filter and NIR $K$ band image is shown in Fig. 5 . HH 633 is a bow shape object of $\sim 20^{\prime \prime}$ length with a bright head, pointing at PA $350^{\circ}$. This object is located $50^{\prime \prime}$ south of IRAS $20591+5214$. On the NIR images IRAS 20591+5214 is a nebulous object with a stellar core. It is also detected in the 2MASS $K$ band image. We are led to suggest that IRAS $20591+5214$ is the source of this flow because of its nebulous nature and because the bow of $\mathrm{HH} 633$ is pointed away from it.

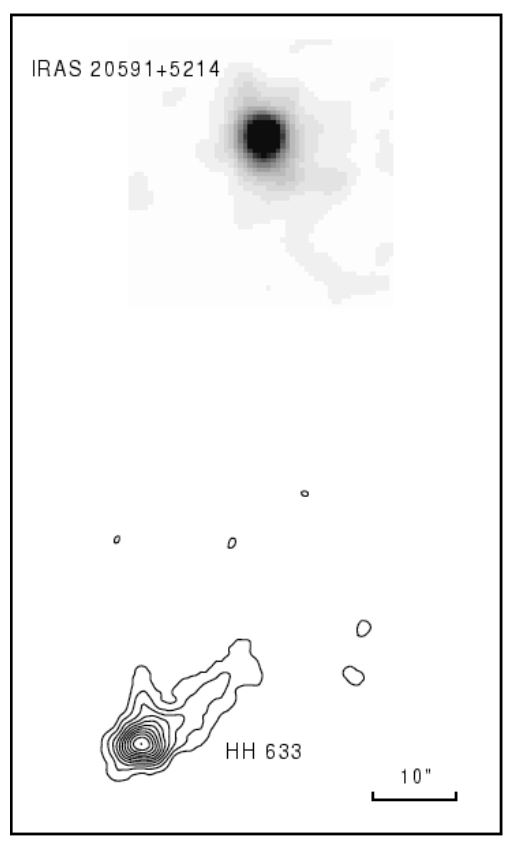

Fig. 5. HH633 in [SII] emission and IRAS 20591+5214 in $K$ band

\subsubsection{HH634-flow}

Figure 6 displays the HH 634 flow at three wavelengths. The top panel displays the $\mathrm{H}_{2} \quad 1-0 \mathrm{~S}(1)$ line, the middle panel 


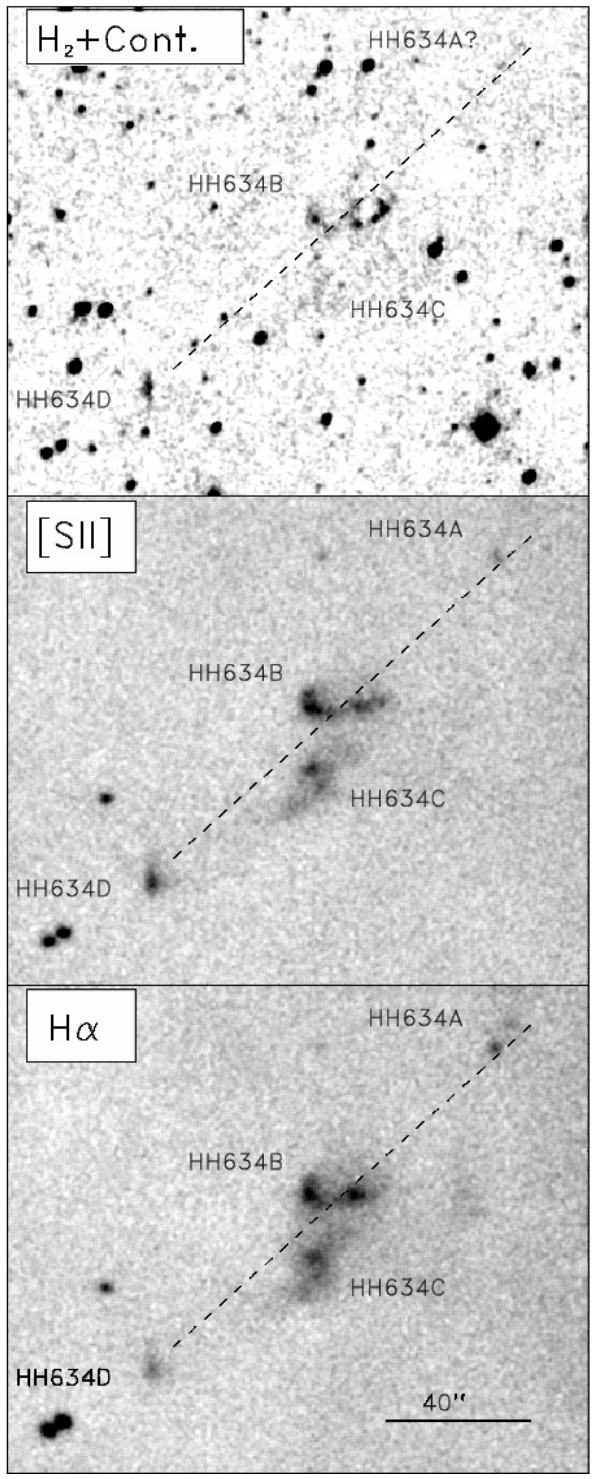

Fig. 6. HH634-flow in $\mathrm{H}_{2}+$ Con., [SII] and $\mathrm{H} \alpha$ lines. The dashed line shows the probable direction of the flow. North is up and east is left.

the [SII] line and the lower one presents the $\mathrm{H} \alpha$ distribution. According to the optical images the HH 634 flow is an elongated structure which consists of $4 \mathrm{HH}$ knots extending towards the south-east. It should be noted that knots B and D are clearly bow-shaped, oriented towards the south-east direction. There is no clear detection of HH 634A in the NIR. The dashed line on all three panels indicates the flow direction as suggested by the knot alignment and individual bow features. There is no clue as to the source of this outflow. However, it is worth mentioning that the knot A in RNO 127 lies near to the probable direction of the HH 634 outflow, but with the opposite orientation.

\subsection{7. $\mathrm{HH} 635$}

This is another chain of $\mathrm{HH}$ objects lying on the faint emission which is traced from the central source of the bright IR nebula in the direction opposite to HH 629 (north-east) and coincides with its axis. HH 635A is the brightest knot also observed in
$\mathrm{H}_{2}$, i.e. despite being in a relatively unobscured region, molecular hydrogen is still present. These objects can be considered as a possible counter flow from the IR source, opposite to the HH 629 system. In this case the flow is "S-shaped" like the HH 315 flow (Reipurth et al. 1997) and we observe a giant bipolar outflow of $2.2 \mathrm{pc}$ in length.

\subsection{Reflection nebulae associated with outflow sources}

\subsubsection{IR Nebula}

During our first imaging observations near RNO 127 we noted the existence of a nearby conspicuous reflection nebula, which was absent on the DSS charts. We called it the IR Nebula because of its brightness in the red-infrared range. In Fig. 7 we present $I$ band, [SII] and $K$ band images of this reflection nebula. One can clearly see (in $I$ band) that in the optical object possesses a sinusoidal morphology, which is completely different from its appearance in the $K$ band. Though the central part of the IR Nebula appears extremely bright in the near infrared, it is not visible in the 2MASS survey $K$ band image, obtained in 1999 and since brightened at least by four magnitudes. This leads us to conclude that its source star recently underwent brightening. Data obtained at different epochs clearly demonstrate the rapid changes in the structure of the nebula, caused probably by propagation of radiation from the central source. It will be interesting to continue monitoring of this object to detect if it has undergone a FUOri-type outburst.

\subsection{2. $\mathrm{CN} 1$ and $\mathrm{HH} 632$}

To the north of RNO 127, a small reflection cometary nebula, connected to IRAS $20590+5221$, was discovered. In the upper part of Fig. 8 [SII] and $I$ band images of the object are presented. On the [SII] image of CN 1 one can clearly see the compact condensation just to the north of the central star, which is absent on the $I$ wide band image. This could be a short emission jet or rather the HH knot which is designated here as HH 632.

\subsection{3. $\mathrm{CN} 2$ and $\mathrm{HH} 630$}

This is a small fan-like reflection nebula to the South-West of RNO 127, associated with the well-collimated jet (HH 630), which is seen only in $\mathrm{H} \alpha$. The morphology of the object is presented in the lower part of Fig. 8. The length of the jet is about $35^{\prime \prime}$, and it is terminated by a faint knot shown with an arrow (lower left panel of Fig. 8).

\subsection{4. $\mathrm{CN} 3$ and $\mathrm{HH} 628$}

Yet another cometary reflection nebula is shown in Fig. 9. In the apex of the nebula the bright star is located which lies inside the error ellipse of the IRAS $20585+5222$ source (see Table 3 ). In about $2^{\prime}$ to the south-east of the CN 3 HH 628 is seen (see left panel of the Fig. 9). It is presumably associated with $\mathrm{CN} 3$, being along its axis. 


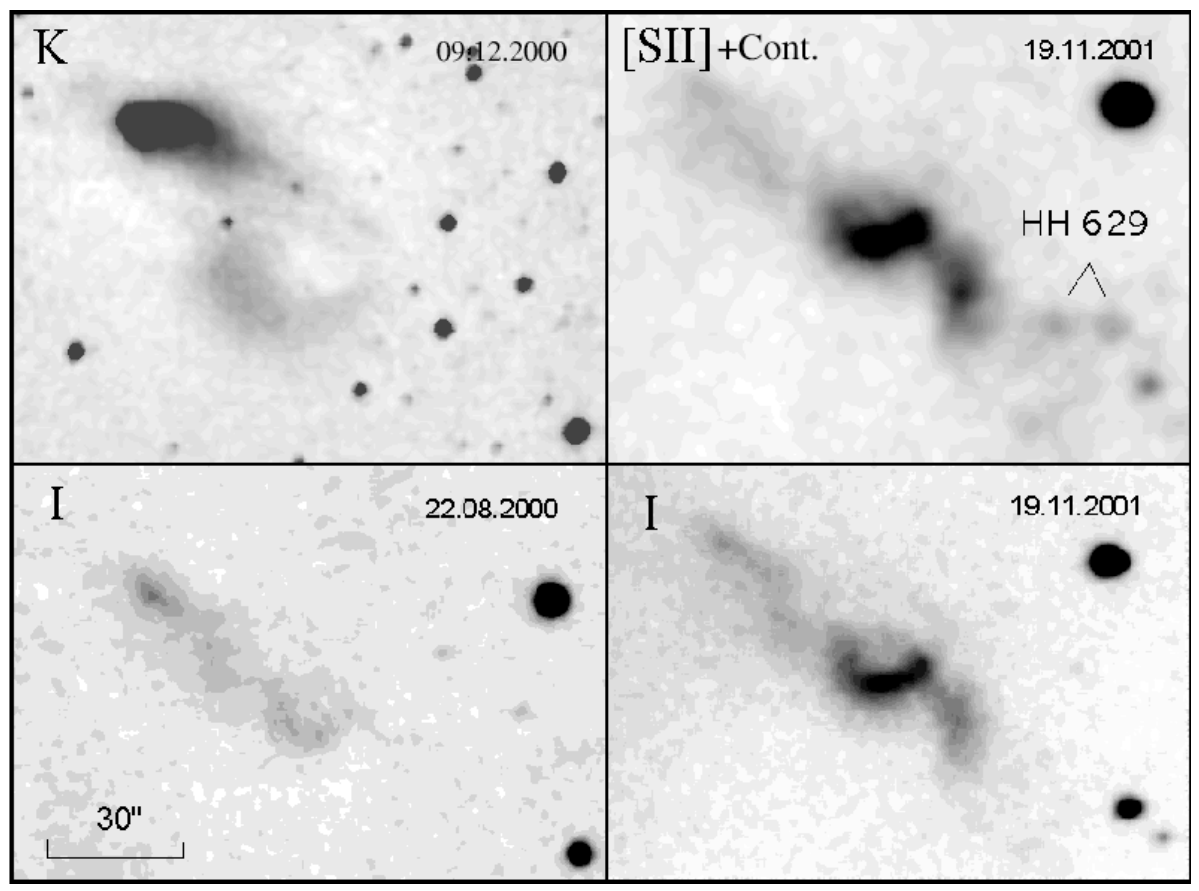

Fig. 7. The appearance of the IR Nebula at various epochs in $I,[\mathrm{SII}]$ and $K$ bands. North is up and east is left.

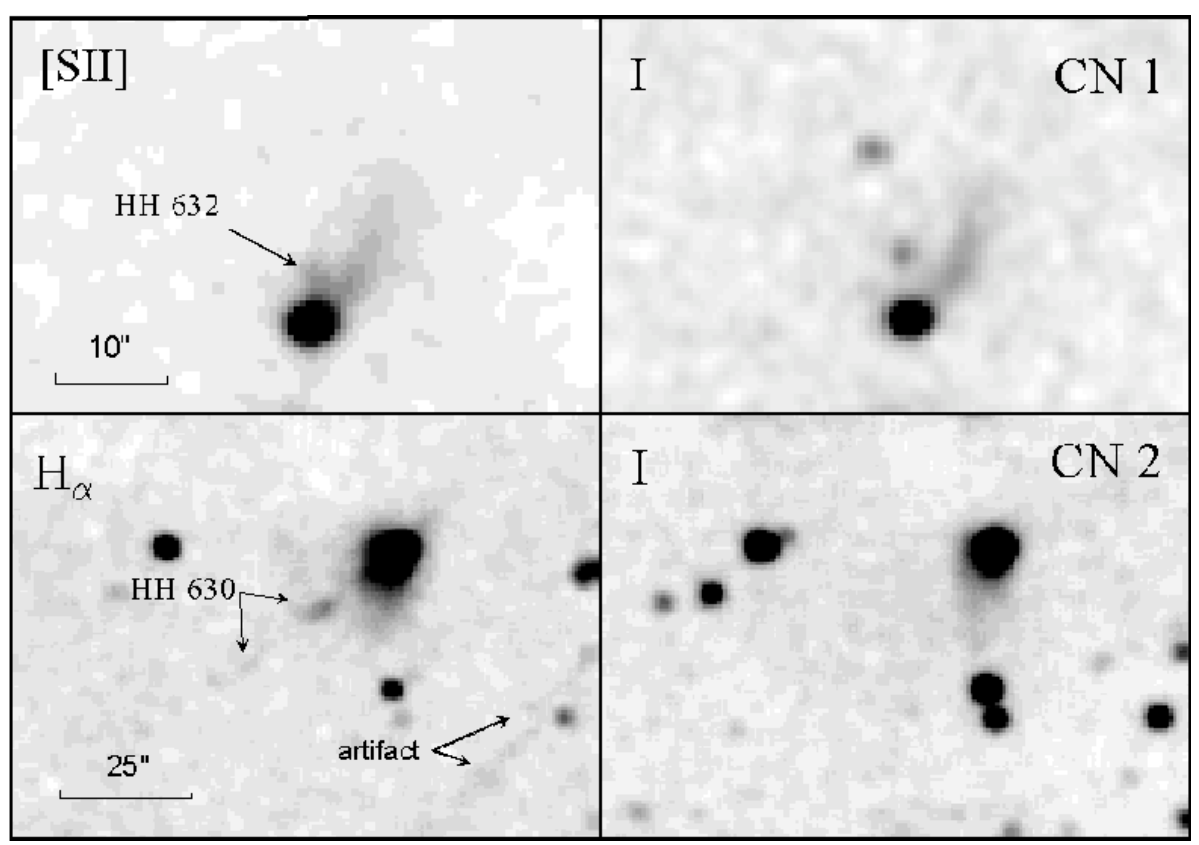

Fig. 8. Upper row: CN 1 - cometary nebula with jet emanating from it (named HH 632). The left panel is the [SII] band image and the right panel is the $I$ band image. Lower row: $\mathrm{CN} 2$ - cometary nebula with HH 630 jet. The left panel is $\mathrm{H} \alpha$ band image, right $-I$ band.

\section{Summary}

We have explored a region in Cygnus in which star formation phenomena appear extensively. The existence of numerous optical and near-infrared outflows suggests that there are several cores in which there is on-going star formation.

Several of the driving sources of $\mathrm{HH}$ flows have been identified with optical objects. All of them are connected to cometary nebulae, oriented along the probable axes of the flows. In one case the nebula contains also a well collimated jet.
The newly-appearing reflection nebula near the significantly brightened stellar source should be especially mentioned. This object deserves further monitoring in both optical and infrared wavelengths.

The $\mathrm{HH}$ objects and $\mathrm{H}_{2}$ knots can be divided into several definite and presumable outflow systems and jets. The brightest $\mathrm{HH}$ object in the region, $\mathrm{HH} 448$, consists of shocked molecular and atomic material. Its structure and excitation is proposed to be the result of the superposition of at least two outflows. A very similar structure has been found in $\rho$ Ophiuchus 


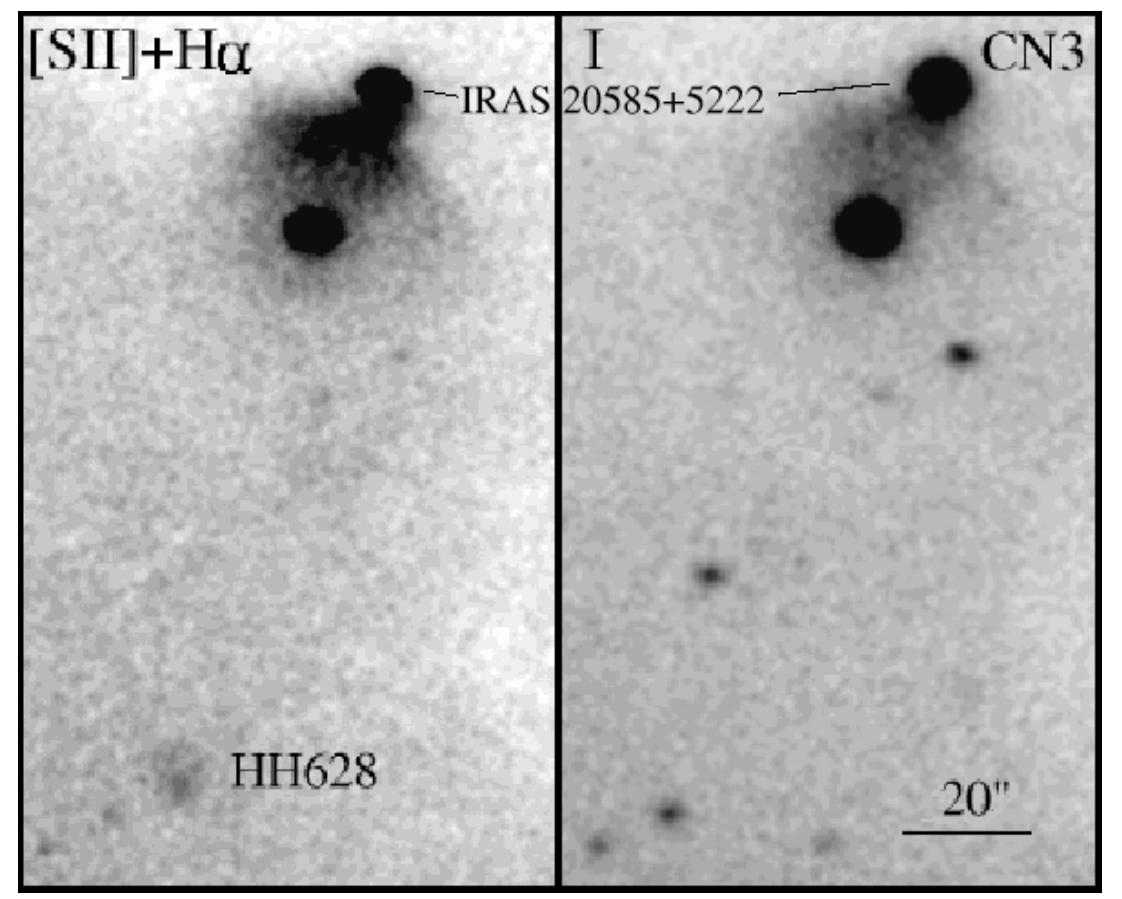

Fig. 9. CN 3 cometary nebula near IRAS $20585+5222$ and $\mathrm{HH} 628$, seen in [SII]+H $\alpha$ filter (left) and in $I$ band (right).

by Grosso et al. (2001), where two outflows appear superimposed. The future search for proper motions will be revealing, e.g. to find if the bow-shaped knot HH448 A is the opposite lobe of a bipolar outflow, the south-eastern lobe of which is represented by $\mathrm{HH} 634$.

One should note also the existence of a possible giant "S-shape" outflow system with a projected length of $2.2 \mathrm{pc}$, represented by the chain of knots in HH 635, lying on the faint emission tail, and by HH 629. This flow coincides with the axis of the IR nebula and, therefore, could be produced by the variable stellar source in the head of the nebula.

Several density enhancements in the cloud are evident. The strongest $100 \mu \mathrm{m}$ emission is from the CN 2 region where the swept-back appearance suggests external compression and the possibility of triggered star formation. The lack of detected $\mathrm{HH}$ objects in the region further to the south-west of this clump is then explained as due to a lack of dense material. In support, we note that only two $\mathrm{HH}$ objects are detected exclusively in the $\mathrm{H}_{2}$ 1-0 S(1) line, NIR2 and NIR3, and these both lie within the $\mathrm{CN} 2$ core region. Hence, the otherwise good correspondence of NIR and optical knots suggests a quite low optical extinction throughout most of the region rather than the selected detection of outflows near the cloud surface.

Acknowledgements. We are grateful to our referee C. Aspin, whose comments and suggestions have improved the presentation of this paper. This project was mainly supported by INTAS grant 00-00287. Also we would like to acknowledge the data analysis facilities provided by the Starlink Project which is run by CCLRC/Rutherford Appleton Laboratory on behalf of PPARC. In addition, the following Starlink packages have been used: CCDPACK, KAPPA and GAIA.

\section{References}

Aumann, H. H., Fowler, J. W., \& Melnyk, M. 1990, AJ, 99, 1674
Beichman, C. A., Neugebauer, G., Habing, H. J., Clegg, P. E., \& Chester, T. J. 1988, NASA Ref. Publ., 1190, 1

Beuther, H., Schilke, P., Sridharan, T. K., et al. 2002, A\&A, 383, 892

Bizenberger, P., McCaughrean, M. J., Birk, C., Thompson, D., \& Storz, C. 1998, Proc. SPIE, 3354, 825

Cohen, M. 1980, AJ, 85, 29

Dame, T. M., Hartmann, D., \& Thaddeus, P. 2001, ApJ

Devine, D., Reipurth, B., Bally, J., in Low Mass Star Formation - from Infall to Outflow, IAU Symp. 182 (poster Proc.), 91

Dobashi, K., Bernard, J.-P., \& Fukui, Y. 1996, ApJ, 466, 282

Dobashi, K., Bernard, J., Yonekura, Y., \& Fukui, Y. 1994, ApJS, 95, 419

Garcia-Lario, P., Manchado, A., Pych, W., \& Pottasch, S. R. 1997, A\&AS, 126, 479

Grosso, N., Alves, J., Neuhäuser, R., \& Montmerle, T. 2001, A\&A, 380, L1

Hartigan, P., Morse, J. A., \& Raymond, J. 1994, ApJ, 436, 125

Heathcote, S., Morse, J. A., Hartigan, P., et al. 1996, AJ, 112, 1141

Joint IRAS Science Working Group 1988, IRAS Point Source Catalog (1988)

Melikian, N. D., \& Karapetian, A. A. 2001, Astrophysics, 44, 216

Melikian, N. D., Karapetian, A. A., Hakhverdian, L. G., \& Karapetian, A. T. 1996, Astrophysics, 39, 115

Motte, F., \& André, P. 2001, A\&A, 365, 440

Movsessian, T., Boulesteix, J., Gach, J.-L., \& Zaratsian, S. 2000, Baltic Astron., 9, 652

Reipurth, B., \& Sandell, G. 1985, A\&A, 150, 307

Reipurth, B., \& Aspin, C. 1997, AJ, 114, 2700

Reipurth, B., Bally, J., \& Devine, D. 1997, AJ, 114, 2708

Reipurth, B., \& Bally, J. 2001, ARA\&A, 39, 403

Smith, M. D., Khanzadyan, T., \& Davis, C. J. 2003, MNRAS, 339, 524

Smith, M. D., Suttner, G., \& Zinnecker, H. 1997a, A\&A, 320, 325

Suttner, G., Smith, M. D., Yorke, H. W., \& Zinnecker, H. 1997, A\&A, 318,595

Wu, J., Wang, M., Yang, J., Deng, L., \& Chen, J. 2002, AJ, 123, 1986 